\title{
Relaxation phenomena of electrical signal emissions from rock following application of abrupt mechanical stress
}

\author{
Dimos Triantis ${ }^{1, \star}$, Filippos Vallianatos ${ }^{2}$, Ilias Stavrakas ${ }^{1}$, George Hloupis ${ }^{1}$ \\ ${ }^{1}$ Technological Educational Institute of Athens, Laboratory of Electric Characterization of Materials and Electronic Devices, Athens, Greece \\ ${ }^{2}$ Technological Educational Institute of Crete, Laboratory of Geophysics and Seismology, Crete, Greece
}

\author{
Article history \\ Received July 16, 2011; accepted February 13, 2012. \\ Subject classification: \\ Mineral physics and properties of rocks, Magnetic and electrical methods, Rock fracture, Mechanical stress, PSC, Amphibolite.
}

\begin{abstract}
The emission of electrical signals during application of mechanical stress to brittle geo-materials (the so-called pressure-stimulated current; PSC) can provide significant information regarding the mechanical status of a studied rock sample. PSCs originate as a result of the opening of cracks and microfractures in rock. In this study, such electrical signal emissions are detected and studied when rock samples are subjected to step-wise mechanical stress, increased from low stress levels $\sigma_{L}$ up to higher stress levels $\sigma_{H}$. This increase is performed at high stress rates and consequently the stress is maintained practically constant for a long period. During this time, the applied stress reaches its maximum value, and the emitted PSC decays gradually and relaxes back to a minimum value. The conducted experiments suggest that the characteristics of the relaxation processes of the PSC depend directly on the high level of the applied stress that is maintained constant after the application of each stress step. Analysis of the macroscopic parameters that characterize the relaxation phenomenon of the PSC provides clear information regarding the proximity of the applied stress to the fracture limit of the rock sample.
\end{abstract}

\section{Introduction}

Laboratory experiments have shown that electromagnetic [Ogawa and Miura 1985, Molchanov and Hayakawa 1995, Nitsan 1997] and electrical [Hadjicontis and Mavromatou 1994, Freud 2002, Vallianatos et al. 2004, Eccles et al. 2005, Baddari et al. 2011] signals are emitted during the application of varying mechanical stress to brittle materials. These signals arise as a result of the nucleation and propagation of micro-fracture events. Several physical mechanisms have been proposed to interpret the emission of electrical signals when a material is subjected to mechanical stress. These include: piezoelectric effects [Nitsan 1997], electrokinetic effects [Ishido and Mizutani 1981], emission of electrons [Brady and Rowell 1986], point defects [Varotsos and Alexopoulos 1986], and motion of charged dislocations [Molchanov and Hayakawa 1995, Vallianatos and Tzanis 1999a, Vallianatos and Tzanis 1999b, Vallianatos et al. 2004].
In recent laboratory experiments, PSC signals have been recorded following various experimental modes relating to applied mechanical stress, and for series of brittle material samples, including marble [Anastasiadis et al. 2004, Stavrakas et al. 2004, Anastasiadis et al. 2007], amphibolite [Triantis et al. 2007], and materials based on cement [Kyriazopoulos et al. 2010, 2011a, 2011b].

The methodology applied to record PSC emissions incorporates connection of an ultra-sensitive electrometer to capture the electrical signals emitted, using a pair of goldplated electrodes mounted collaterally on the stressed sample. This technique has been described as the PSC technique [Anastasiadis et al. 2004]. To date, studies of electrical signals recorded using the PSC technique have led to a number of findings. Studies on rock samples [Stavrakas et al. 2004] and on cement-based materials [Kyriazopoulos et al. 2011a] have shown that upon application of a monotonic axial stress at a constant stress rate, PSC emissions are detected when the mechanical stress applied becomes greater than the yield stress value, and the sample enters the non-linear region of its stress-strain behavior. During this experimental procedure, the PSC signal reaches its maximum value slightly before the failure of the sample.

Other studies have shown that the emitted PSCs are clearly related to mechanical strain, which can be used as a damage indicator. Considering the memory phenomenon that appears in acoustic emissions during cyclic mechanical loading (i.e. the Kaiser effect), similar phenomena have been reported in terms of PSC emissions that have shown that repetitive mechanical loading cycles generate gradually lower PSC emissions [Anastasiadis et al. 2007].

It has been shown experimentally and supported theoretically that when a brittle geomaterial is subjected to gradually increasing mechanical stress at a constant rate up to its fracture, the total electric charge that is released is not affected by the applied stress rate [Triantis et al. 2006]. 
Using the above-mentioned experimental technique, it was shown that when the mechanical stress applied to a sample exceeds the yield stress value, the electric charge released and the mechanical strain are linearly related [Triantis et al. 2008]. The experimental verification of this linear relationship appears to support the theoretical predictions of the moving charged dislocation model. This supports the existence of a proportionality between the electric current density and the strain rate, which provides a good interpretation of the underlying mechanisms of electrical signal emission [Slifkin 1993, Vallianatos and Tzanis 1998, Tzanis and Vallianatos 2001].

A power-law for the time-to-failure relationship that is based on irreversible thermodynamics describes the cumulative energy release of PSC as failure approaches. All of the aforementioned similarities between acoustic emissions and seismicity, and also the form of the power laws, suggest connections with fracture phenomena at much larger scales This implies that a basic general mechanism is 'actively hidden' behind all of these phenomena [Vallianatos and Triantis 2008]. Furthermore, interesting results have been extracted from preliminary experiments of the application of sequential abrupt stress that increases up to the failure of samples of rock [Vallianatos et al. 2004] and cement-based materials [Kyriazopoulos et al. 2011a]. Comparison of PSC signals recorded for these two types of samples has shown clear similarities regarding their qualitative macroscopic characteristics, but significant differences regarding their quantitative characteristics: PSC emissions recorded from cement-based materials are significantly greater than those from rock materials.

In the present study, high-rate sequential stress steps were applied to amphibolite samples and their corresponding PSC emissions were recorded. As it is known that the PSCs emitted are related to the damage caused in the bulk sample, an attempt was made to correlate the qualitative characteristics of the PSC emissions, of the applied mechanical stress, and of the consequent damage that was caused to the sample. The main objective of this experimental study was to determine the upcoming fracture of the samples through the study of the macroscopic characteristics of the electrical signals.

\section{Amphibolite: the samples}

The samples that were used in the present study were of fine-grained amphibolite rock. The materials were extracted from a depth of ca. $6.0 \mathrm{~km}$ at the drilling site of the German KTB continental deep drilling program [Lich et al. 1992]. The compositions of the specific types of amphibolite included the dominant minerals of hornblende, plagioclase and garnet, together with minor quartz and biotite. These compositions were supplemented by Ti-phase ilmenite (1\%$3 \%$ ), opaques, K-field spar, apatite, and some zircon. The porosity of the samples was less than $1 \%$ vol. [Lich et al. 1992, Nover et al. 1995].

The samples used were shaped in a cylindrical form of $25-\mathrm{mm}$ diameter and $60-\mathrm{mm}$ height. The fracture limit was calculated to range between $49 \mathrm{MPa}$ and $53 \mathrm{MPa}$.

\section{Experimental techniques}

PSC signals were detected when the samples were under continuous mechanical stress that varied from low to a high levels, when close to fracture. The axial stress was increased through abrupt stress steps. Specifically, a procedure of seven abruptly increasing stress steps was applied. This technique has been termed the 'abrupt ratestep stress' technique [Kyriazopoulos et al. 2011a], and can be described in detail as follows.

The temporal description of a stress $\sigma(t)$ can be described with the following mathematical expression:

$$
\sigma(t)=\left[\begin{array}{ccc}
\sigma_{L}=\text { constant } & \text { for } & t<t_{L} \\
\sigma_{L}+b\left(t-t_{L}\right) & \text { for } & t_{L} \leq t \leq t_{k}+\Delta t=t_{H} \\
\sigma_{H}=\sigma_{L}+\Delta \sigma & \text { for } & t>t_{H}
\end{array}\right.
$$

The sample is taken to an initial constant uniaxial stress level, $\sigma_{L}$. The application of this stress at this $\sigma_{L}$ value lasts for a time interval $t_{L}$ whereby the PSC electrical signal can attain its minimum possible value (background). Then, a stress increase of $\Delta \sigma$ follows at an optimal constant increase rate of $b=\Delta \sigma / \Delta t$, with the sample taken to a higher stress level $\sigma_{H}$. At each stress step, the pressure increase from the low level $\sigma_{L}$ to the higher level $\sigma_{H}$ takes place at a fast rate (ca. 3.5 MPa/s). The sample remains at this higher level $\sigma_{H}$ for considerable time, so that the PSC signal can again relax to its lowest possible level.

The application of sequential stress steps (whereby the level $\sigma_{H}$ of one step then becomes the $\sigma_{L}$ of the next stress step, and so on), leads to the development and detection of PSC signals that reflect the whole range of the mechanical behavior of each sample.

The experimental set-up of the mechanical parts that were used to apply the uniaxial compressional stress has been presented in detail in previous studies [e.g. Triantis et al. 2007], and only the essential details are given here (see Figure 1). The electrical signal is detected by recording weak electric currents using a sensitive electrometer. The electric current is captured through a pair of gold-plated electrodes that are attached to the sides of the sample that is under mechanical stress. Teflon sheets of 2-mm thickness are placed on both the upper and lower surfaces of each sample, as electrical insulators. To perform the electrical measurements, a sensitive programmable electrometer was used (Keithley 6514). The experiments were conducted in a Faraday shield to prevent the measurements from being affected by electrical noise. 


\section{Experimental results and discussion}

Several experiments were carried out using the abovedescribed experimental technique and representative experimental results are presented here. A sequence of seven stress steps was applied (see Figure 2a), starting from a low axial stress level of $6 \mathrm{MPa}$ (the low-stress state $\sigma_{L}$ of the first stress step) up to $49 \mathrm{MPa}$, which was close to the fracture limit $\left(\sigma_{F}\right)$ of the samples. It can be noted that the high stress level of the seventh stress step was 48.8 $\mathrm{MPa}$. The attempted application of an eighth stress step was not accomplished, as the sample failed at $49.35 \mathrm{MPa}$ (0.55 MPa higher than the stress level $\sigma_{H}$ of the seventh stress step).

The exact values of these $\sigma_{L}$ and $\sigma_{H}$ levels of each stress step for this sample are given in Table 1 . Figure $2 \mathrm{~b}$ shows the temporal recording of the PSC signals with respect to the sequence of the seven abrupt stress steps.

During each stress step, when the abrupt transition from $\sigma_{L}$ to $\sigma_{H}$ occurred, the consequent PSC excitation was recorded. The PSC signals show a peak, practically at the moment $t_{0}$ where the stress reaches the $\sigma_{H}$ levels. The PSC $\mathrm{Pax}_{\max }$ of each of these stress steps are given in Table 1. Then the PSC signals decreased to reach the relaxation levels. The mean values of these relaxation levels are referred to as $\overline{P S C_{\text {min }}}$. These values corresponding to each stress step as the $\overline{P S C_{\min }}$ were calculated and are given in Table 1.

During the periods after the stabilization of the stress at the high level at each stress step, the PSC relaxed following the exponential law:

$$
P S C\left(t^{\prime}\right)=P S C_{\max } \cdot \exp \left(-\frac{t^{\prime}}{\tau}\right) \quad \text { for } \quad 0 \leq t^{\prime} \leq t_{e} \quad(1)
$$

where $t^{\prime}=t-t_{0}, \tau$ is a relaxation time constant, and $t_{e}$ is a characteristic time parameter up to which the PSC signal obeys the exponential law of relaxation described in Equation (2).

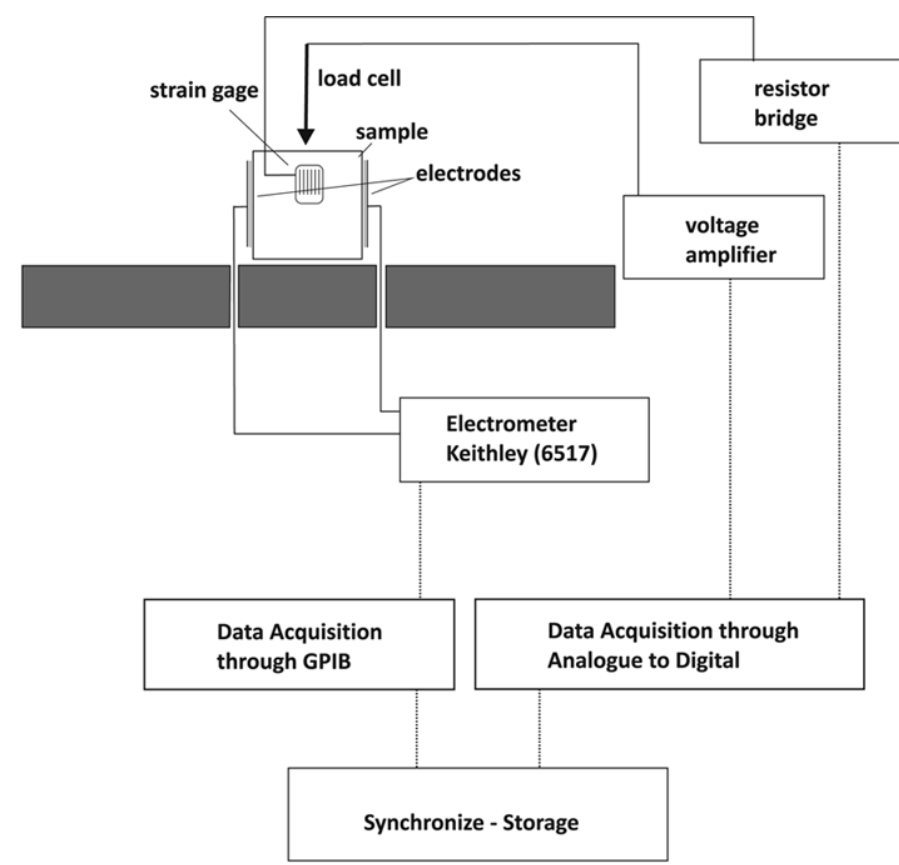

Figure 1. The experimental apparatus, as installed to capture the PSCs emitted during the application of the mechanical stress.

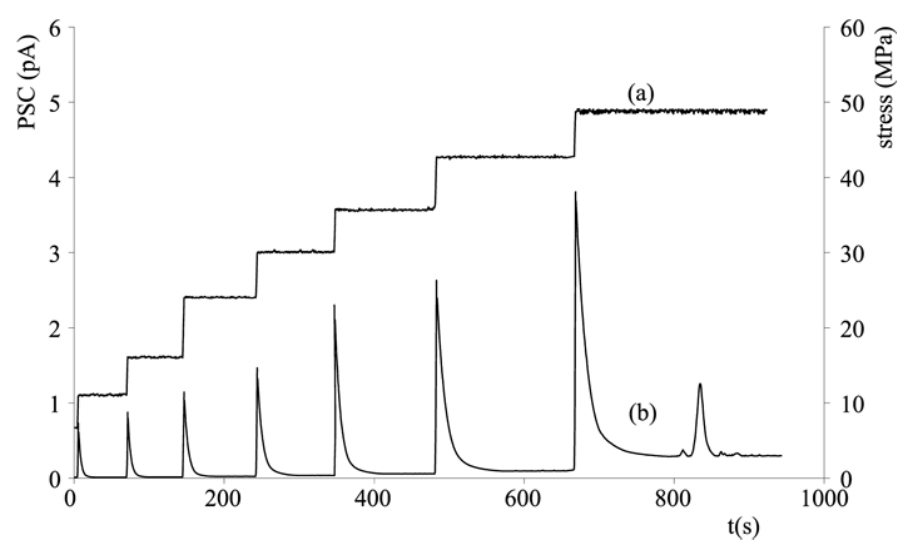

Figure 2. Recording (a): The sequence of the seven stress steps. Recording (b): The corresponding temporal variations in the PSC emissions recorded.

\begin{tabular}{|c|c|c|c|c|c|c|c|}
\hline Step & $\sigma_{L}(M p A)$ & $\sigma_{H}(M p A)$ & $\begin{array}{l}\sigma_{F}: 49.35 \mathrm{MPa} \\
\sigma_{F}-\sigma_{H}(\mathrm{MPa})\end{array}$ & $\begin{array}{c}\mathrm{PSC}_{\text {max }} \\
(\mathrm{pA})\end{array}$ & $\tau(\mathbf{s})$ & $t_{e}(s)$ & $\begin{array}{r}\overline{P S C_{\text {min }}} \\
(\mathrm{pA})\end{array}$ \\
\hline 1 & 6.69 & 11.05 & 38.30 & 0.73 & 3.20 & 7.4 & 0.011 \\
\hline 2 & 11.13 & 16.07 & 33.28 & 0.87 & 3.90 & 8.1 & 0.014 \\
\hline 3 & 16.07 & 24.03 & 25.32 & 1.14 & 5.30 & 10.2 & 0.022 \\
\hline 4 & 23.99 & 30.08 & 19.27 & 1.47 & 7.00 & 12.1 & 0.035 \\
\hline 5 & 30.08 & 35.66 & 13.69 & 1.90 & 8.10 & 13.4 & 0.057 \\
\hline 6 & 35.82 & 42.70 & 6.65 & 2.67 & 12.10 & 19.0 & 0.105 \\
\hline 7 & 42.71 & 48.80 & 0.55 & 3.81 & 16.74 & 24.5 & 0.301 \\
\hline
\end{tabular}

Table 1. The $\sigma_{L}$ and $\sigma_{H}$ levels of the seven sequential stress steps, with the corresponding values of the macroscopic characteristics of the PSC signals recorded. 


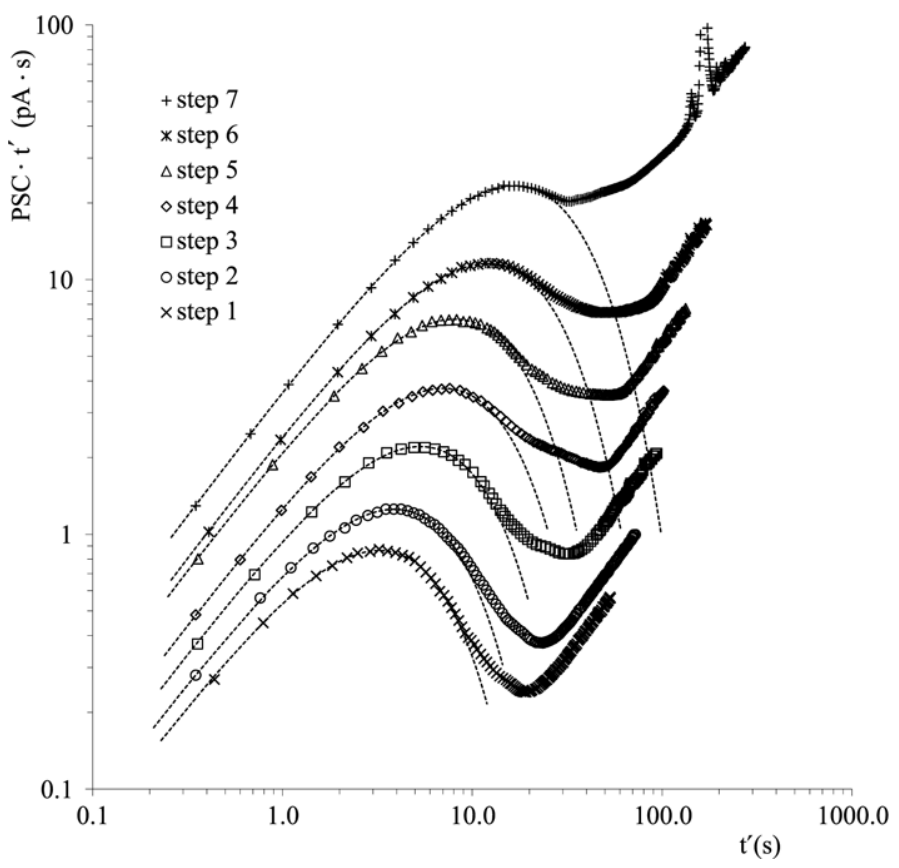

Figure 3. The seven PSC signals with respect to time $t^{\prime}\left(\left[\operatorname{PSC}\left(t^{\prime}\right)\right] \cdot t^{\prime}\right.$ vs $\left.t^{\prime}\right)$. The time scale $t^{\prime}=t-t_{0}$, where $t_{0}$ is the time when the PSC signal reaches its maximum value. The dotted curves correspond the the best exponential fitting according to Equation (2) The PSC signals deviate from the exponential law after a time $t_{e}$ which is characteristic for each stress step.

This exponential relaxation of the PSC signals during these periods can be verified from the plot of $\left[\operatorname{PSC}\left(t^{\prime}\right)\right] \cdot t^{\prime}$ versus $t^{\prime}$ for each of the seven PSC recordings for each corresponding
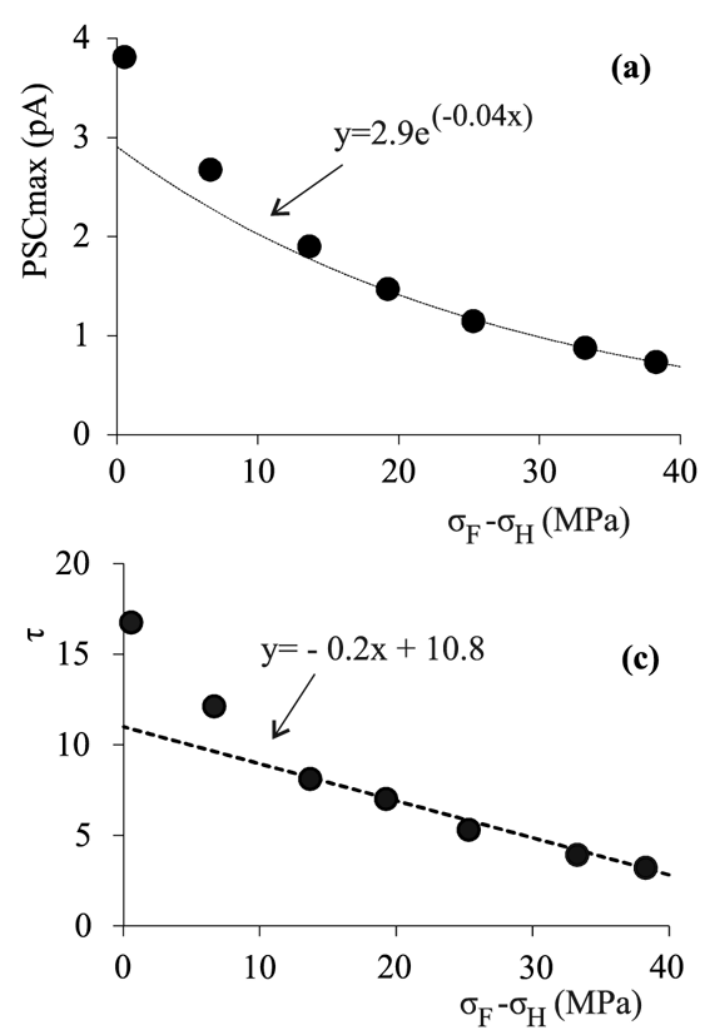

stress step (see Figure 3).

The value $t^{\prime}$ at which the maximum of the function $\left[\operatorname{PSC}\left(t^{\prime}\right)\right] \cdot t^{\prime}$ is reached corresponds to the time constant $\tau$. Through best fitting to Equation (2) (Figure 3, dotted curves), the times $t_{e}$ can be estimated for each of these seven PSC signals. These values of $\tau$ and $t_{e}$ are given in Table 1.

Both the presentation of the PSC signal recordings and the recorded values given in Table 1 lead to the conclusion that the macroscopic characteristics of the PSC signal depend on the high level $\sigma_{H}$ of each stress step, such as for $P S C_{\max }, \overline{P S C_{\min }}$ and the relaxation parameters $\left(\tau\right.$ and $\left.t_{e}\right)$.

Figure 4 shows the behaviour of the above-mentioned quantities as functions of $\sigma_{F}-\sigma_{H}$. It is important to note the following: $P S C_{\max }$ and $P S C_{\min }$ of the PSC signals appear to be related to $\sigma_{F}-\sigma_{H}$ through an empirical law of the form: $\mathrm{A} \cdot \exp \left[-\mathrm{a}\left(\sigma_{F}-\sigma_{H}\right)\right]$, where $\mathrm{A}$ and a are constants. This empirical law appears to be validated when $\sigma_{F}-\sigma_{H}$ has values greater than approximately $10 \mathrm{MPa}$. When $\sigma_{F}-\sigma_{H}$ $<10 \mathrm{MPa}$, the sample is practically in the nonlinear region regarding its mechanical properties, and the values of $P S C_{\max }$ and $\overline{P S C_{\text {min }}}$ gradually deviate from this exponential law. This behavior arises as the crack formation and propagation that is caused by the abrupt stress increase are facilitated by the level of the applied mechanical stress (i.e. higher than the yielded stress), which is high enough to produce further cracks and extend the existing cracks. That is the reason the recorded

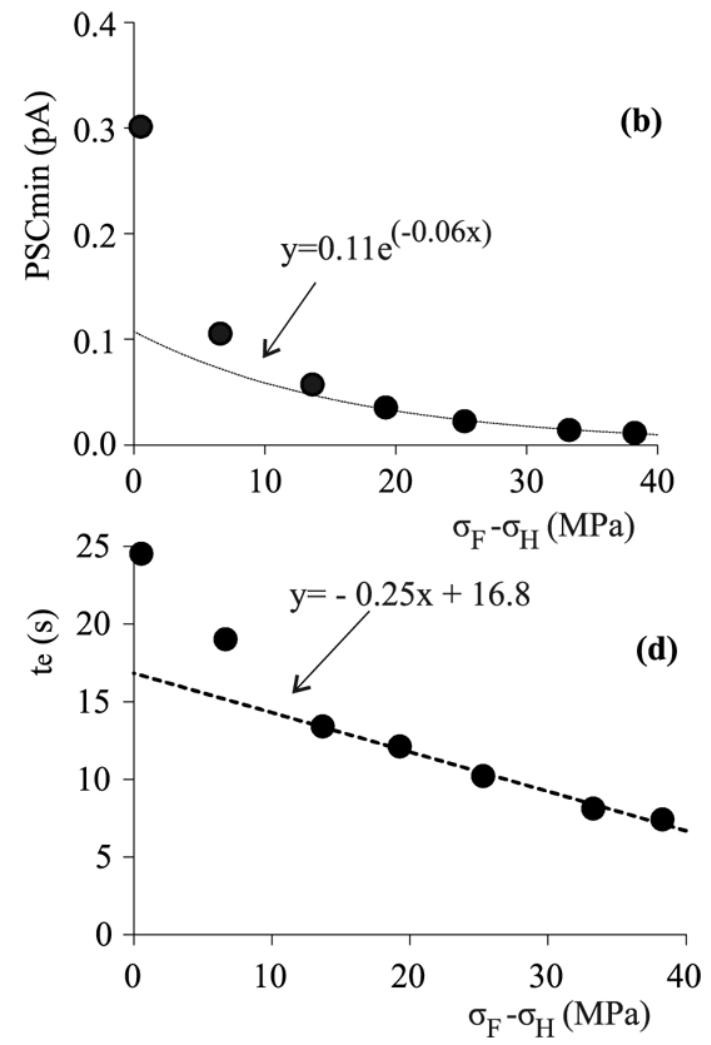

Figure 4. (a) $P S C_{\max }$, (b) $\overline{P S C_{\min }}$, (c) $\tau$ and (d) $t_{e}$ of the seven stress steps with respect to $\sigma_{F}-\sigma_{H}$. 


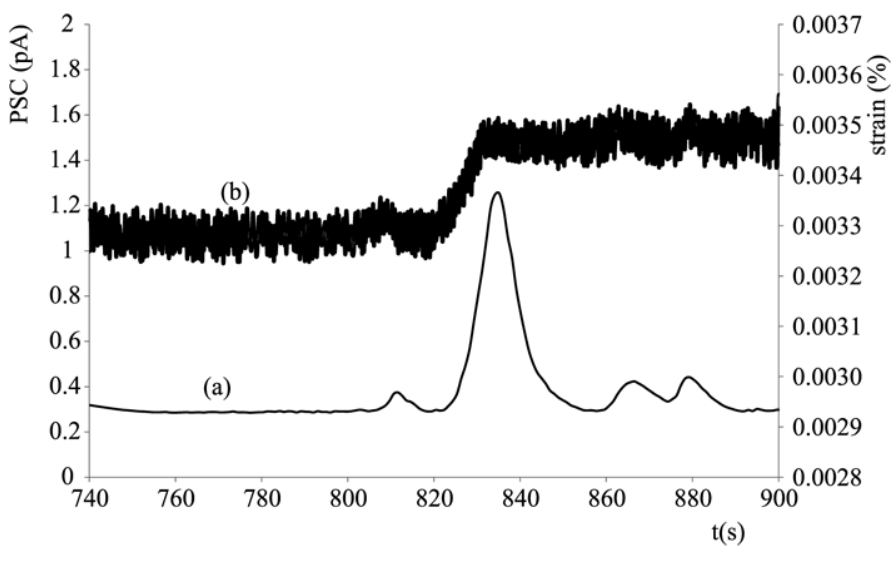

Figure 5. Recording (a): PSC excitation when the amphibolite sample was under high levels of mechanical stress close to fracture $\left(\sigma_{H} \rightarrow \sigma_{F}\right)$. Recording (b): The corresponding behavior of the mechanical strain of the sample. The PSC excitation appears to be directly related to the corresponding abrupt increase in the strain.

PSC shows higher relaxation times and higher $\overline{P S C_{\min }}$.

It is interesting to observe the dependence of the relaxation time constant $\tau$ on $\sigma_{F}-\sigma_{H}$. This constant follows a linear law for values of $\sigma_{F}-\sigma_{H}>10 \mathrm{MPa}$, while for $\sigma_{F}-\sigma_{H}$ $<10 \mathrm{MPa}$ the corresponding values significantly deviate from this linearity and increase as $\sigma_{F}-\sigma_{H}$ reaches a minimum. Similar behavior is seen for the characteristic time duration $t_{e}$, which is related to the time during which the PSC signal follows the exponential relaxation given by Equation (2).

Finally, there is another interesting observation regarding the study of the PSC signals, especially in the region where the $\sigma_{H}$ of the stress step is close to the fracture limit of $\sigma_{F}$ of the sample. While the PSC signal tends to return to $\overline{P S C_{\text {min }}}$, sudden PSC excitation occurs (in the form of peaks; see Figure 5, recording (a); the peaks appeared between $800 \mathrm{~s}$ and $900 \mathrm{~s}$ ). The appearance of these peaks is related to the fluctuation in the strain rate (see Fig. 5, recording (b)). This has also been observed experimentally for cement samples [Kyriazopoulos et al. 2011a]. The appearance of these PSC peaks under this constant stress regime and following previous step stressing predicts the forthcoming sample failure. The appearance of such sharp peaks at this stage reflect the irreversible accumulation and coalition of macrofractures, to create the final stage of fracture that breaks the sample.

\section{Conclusions}

When conducting laboratory experiments on finegrained amphibolite rock samples using the abrupt rate-step stress technique, electrical signals, known as PSCs, are emitted and can be analyzed. The analysis of these PSCs shows that their macroscopic characteristics, such as the $P S C_{\max }$, the $\overline{P S C_{\min }}$ and their relaxation parameters ( $\tau$ and $t_{e}$ ), depend strongly on the higher level, $\sigma_{H}$, of each stress step.

Thus, this technique can provide information (and is a potential tool) towards rough estimation of fracture limits, although without damaging the sample. This can be achieved for a known type of material (e.g. rock), if systematic calibration is available, through the calculation of the various values of these macroscopic characteristics for a set of stress levels. Consequently, when a mechanical stress step that corresponds to the higher stress level $\sigma_{H}$ is applied to a sample that has never suffered any mechanical stress previously, the macroscopic characteristics of the PSC recorded can lead to estimation of the proximity of $\sigma_{H}$ to the fracture limit, $\sigma_{F}$.

Another conclusion that is reached in the present study is that the development of new PSC current excitations for a rock sample that has relaxed following the application of a practically constant stress indicate the proximity of the applied stress to the fracture limit.

Acknowledgements. This work was supported in part by the THALES Program of the Ministry of Education of Greece and the European Union in the framework of the project entitled "Integrated understanding of seismicity, using innovative methodologies of fracture mechanics along with earthquake and nonextensive statistical physics application to the geodynamic system of the Hellenic Arc (SEISMO FEAR HELLARC)".

\section{References}

Anastasiadis, C., D. Triantis, I. Stavrakas and F. Vallianatos (2004). Pressure stimulated currents (PSCs) in marble samples after the application of various stress modes before fracture, Annals of Geophysics, 47 (1), 21-28.

Anastasiadis, C., D. Triantis and C.A. Hogarth (2007). Comments on the phenomena underlying pressure stimulated currents (PSCs) in dielectric rock materials, J. Mater. Sci., 42, 2538-2542.

Baddari, K., A.D. Frolov, V. Tourtchine and F. Rahmoune (2011). An integrated study of the dynamics of electromagnetic and acoustic regimes during failure of complex macrosystems using rock blocks, Rock Mech. Rock Eng., 44, 269-280.

Brady, B.T., and G.A. Rowell (1986). Laboratory investigation of the electrodynamics of rock fracture, Nature, 321, 448-492.

Eccles, D., P.R. Sammonds and O.C. Clint (2005). Laboratory studies of electrical potential during rock failure, Intl. J. Rock Mech. Min., 42, 933-949.

Freund F. (2002). Charge generation and propagation in rocks, J. Geodyn., 33, 545-572.

Hadjicontis, V., and C. Mavromatou (1994). Transient electric signals prior to rock failure under uniaxial compression, Geophys. Res. Lett., 21, 1687-1690.

Ishido, T., and H. Mizutani (1981). Experimental and theoretical basis of electrokinetic phenomena in rock-water systems and its applications to geophysics, J. Geophys. Res., 86, 1763-1775.

Kyriazopoulos, A., I. Stavrakas, K. Ninos, C. Anastasiadis and D. Triantis (2010). Pressure-stimulated current emissions 
on cement paste samples under repetitive stepwise compressional loadings, Strojniški vestnik - Journal of Mechanical Engineering, 56, 551-556.

Kyriazopoulos, A., C. Anastasiadis, D. Triantis and J.C. Brown (2011a). Non-destructive evaluation of cementbased materials from pressure-stimulated electrical emission - preliminary results, Constr. Build. Mater., 25, 1980-1990.

Kyriazopoulos, A., I. Stavrakas, C. Anastasiadis and D. Triantis (2011b). Study of weak electric current emission on cement mortar under uniaxial compressional mechanical stress up to the vicinity of fracture, Strojniški vestnik Journal of Mechanical Engineering, 57, 235-242.

Lich, S., J. Duyster, G. Godizart, S. Keyssner and H. de Wall (1992). German Continental Deep Drilling Program (KTB) - Geological Survey of the Hauptbohrung 0-6,000 m, KTB Report, 92-2, B1-B83.

Molchanov, O.A., and M. Hayakawa (1995). Generation of ULF electromagnetic emissions by microfracturing, Geophys. Res. Lett., 22, 3091-3094.

Nitsan, U (1997). Electromagnetic emission accompanying fracture of quartz-bearing rocks, Geophys. Res. Lett., 4, 333-337.

Nover, G., A. Heikamp, A. Kontny and A. Duba (1995). The effect of pressure on electrical conductivity of KTB rocks, Surv. Geophys., 16 (1), 63-81.

Ogawa, T. K., and T. Miura (1985). Electromagnetic radiation from rocks, J. Geophys. Res., 90, 6245-6249.

Slifkin, L. (1993). Seismic electric signals from displacement of charged dislocations, Tectonophysics, 224, 149-152.

Stavrakas, I., D. Triantis, Z. Agioutantis, S. Maurigiannakis, V. Saltas and F. Vallianatos (2004). Pressure stimulated currents in rocks and their correlations with mechanical properties, Nat. Hazards Earth Sys., 4, 563-567.

Triantis, D., I. Stavrakas, C. Anastasiadis, A. Kyriazopoulos and F. Vallianatos (2006). An analysis of pressure stimulated currents (PSCs), in marble samples under mechanical stress, Phys. Chem. Earth, 31, 234-239.

Triantis, D., C. Anastasiadis, F. Vallianatos, P. Kyriazis and G. Nover (2007). Electric signal emissions during repeated abrupt uniaxial compressional stress steps in amphibolite from KTB drilling, Nat. Hazards Earth Sys., 7, 149-154.

Triantis, D., C. Anastasiadis and I. Stavrakas (2008). The correlation of electrical charge with strain on stressed rock samples, Nat. Hazards Earth Sys., 8, 1243-1248.

Tzanis, A., and F. Vallianatos (2001). A critical review of ULF electric earthquake precursors, Annals of Geophysics, 44 (2), 23-35.

Vallianatos, F., and A. Tzanis (1998). Electric current generation associated with the deformation rate of a solid: preseismic and co-seismic signals, Phys. Chem. Earth, 23, 933-938.

Vallianatos, F., and A. Tzanis (1999a). A model for the gen- eration of precursory electric and magnetic fields associated with the deformation rate of the earthquake focus, In: M. Hayakawa (ed.), Seismic Atmospheric \& Ionospheric Electromagnetic Phenomena, Terra Scientific Publishing Co., Tokyo.

Vallianatos, F., and A. Tzanis (1999b). On possible scaling laws between electric earthquake precursors (EEPs) and earthquake magnitude, Geophys. Res. Lett., 26, 2013-2016.

Vallianatos, F., D. Triantis, A. Tzanis, C. Anastasiadis and I. Stavrakas (2004). Electric earthquake precursors: from laboratory results to field observations, Phys. Chem. Earth, 29, 339-351.

Vallianatos, F., and D. Triantis (2008). Scaling in pressure stimulated currents related with rock fracture, Physica A, 387, 4940-4946.

Varotsos, P., and K. Alexopoulos (1986). Thermodynamics of Point Defects and their Relation with Bulk Properties, Noth-Holand, Amsterdam, 23-80.

\footnotetext{
${ }^{\star}$ Corresponding author: Dimos Triantis,

Technological Educational Institute of Athens, Laboratory of Electric Characterization of Materials and Electronic Devices, Athens, Greece; email: triantis@teiath.gr.

(C) 2012 by the Istituto Nazionale di Geofisica e Vulcanologia. All rights reserved.
} 